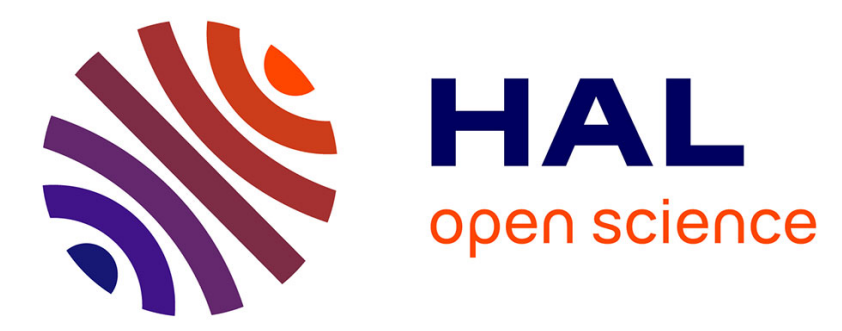

\title{
Optimization of Hybrid Imaging Systems Including Digital Deconvolution in the Presence of Noise
}

Frédéric Diaz, François Goudail, Brigitte Loiseaux, Jean-Pierre Huignard

\section{To cite this version:}

Frédéric Diaz, François Goudail, Brigitte Loiseaux, Jean-Pierre Huignard. Optimization of Hybrid Imaging Systems Including Digital Deconvolution in the Presence of Noise. Imaging Systems (IS), Jun 2010, Tucson, Arizona, United States. pp.IMD4. hal-00752204

\section{HAL Id: hal-00752204 \\ https://hal-iogs.archives-ouvertes.fr/hal-00752204}

Submitted on 15 Nov 2012

HAL is a multi-disciplinary open access archive for the deposit and dissemination of scientific research documents, whether they are published or not. The documents may come from teaching and research institutions in France or abroad, or from public or private research centers.
L'archive ouverte pluridisciplinaire HAL, est destinée au dépôt et à la diffusion de documents scientifiques de niveau recherche, publiés ou non, émanant des établissements d'enseignement et de recherche français ou étrangers, des laboratoires publics ou privés. 


\title{
Optimization of hybrid imaging systems including digital deconvolution in the presence of noise
}

\author{
Frédéric Diaz $^{1,2}$, François Goudail ${ }^{2}$, Brigitte Loiseaux ${ }^{1}$, Jean-Pierre Huignard ${ }^{1}$ \\ ${ }^{1}$ Thales Research \& Technology, 1 Avenue Augustin Fresnel, 91767 Palaiseau CEDEX, France \\ ${ }^{2}$ Laboratoire Charles Fabry de l'Institut d'Optique, CNRS, Université Paris-Sud, Campus Polytechnique, RD 128, 91127 Palaiseau CEDEX, \\ France \\ *Corresponding author : frederic.diaz@thalesgroup.com
}

\begin{abstract}
We address the depth of focus enhancement in hybrid imaging systems, including a phase mask and a deconvolution filter. A final image quality criterion is introduced to optimize and compare different masks.
\end{abstract}

\section{Introduction}

In a classical imaging system, the object is imaged on a sensor by an optical system which should provide an image as good as possible. A post processing step can then be implemented, but the optimization of the optics and the post processing is done separately. In an hybrid imaging system, the optimization of the optics and the post-processing step is performed jointly, and the post processing can correct some aberrations of the optics. By introducing a phase mask into the optical systems, we can obtain an imaging system with a point spread function (PSF) insensitive to the defocus for a given range of object positions. This blurred image is then post processed in order to recover its quality.

Several phase masks can extend the depth of focus, such as the cubic [1] or the exponential phase mask [2]. They depend on parameters that are optimized with respect to an aimed application. In order to optimize them and to compare their performance, we recently proposed a criterion based on the image quality [3] after the post-processing step. This optimization results from a compromise between two antagonistic effects: the variation of the PSF with respect to defocus, whereas the deconvolution filter is unique, and the noise enhancement due to deconvolution, which increases as the imaging system has a low spatial band pass behavior. With usual phase masks, the more invariant to defocus the PSF is, the more they degrade the performance after the post processing.

\section{Definition of the optimization criterion}

We proposed an image quality criterion [3] in order to optimize the parameters of depth of focus enhancing phase masks. It is based on the mean square error between an object $O(r)$ and its estimate $\hat{O}(r)$. The image $I_{\psi}(r)$ of the object through an optical system at a defocus $\psi$ is:

$$
I_{\psi}(r)=h_{\psi}(r) * O(r)+n(r),
$$

where $n(r)$ is the detection noise and $h_{\psi}(r)$ the point spread function of the optical system for a given defocus $\psi$ given by

$$
\psi=\pi R^{2} / \lambda \times\left(1 / d_{O}+1 / d_{I}-1 / f\right),
$$

with $R$ the radius of the aperture, and $f, d_{O}$ and $d_{I}$ respectively the focal length, the object distance and the image sensor plane distance, and the symbol $*$ refers to the convolution operation. The amount of noise in the scene is quantified by the input SNR defined as:

$$
\operatorname{SNR}_{\text {in }}(d B)=10 \log _{10}\left\lfloor\int S_{O O}(v) d v / \int S_{n n}(v) d v\right\rfloor .
$$

The estimate of the object is obtained through a linear deconvolution filter $d(r)$, and is equal to $\hat{O}(r)=d(r) * I_{\psi}(r)$. Assuming that $n(r)$ and $O(r)$ are stationary random processes with power spectral densities $S_{n n}(v)$ and $S_{O O}(v)$, the mean squared error (MSE) between the object and its estimate for a given defocus $\psi$ is: 


$$
\begin{aligned}
M S E_{\psi} & =\left\langle\left|O(r)-d(r) * I_{\psi}(r)\right|^{2}\right\rangle \\
& =\int\left|\tilde{d}(v) \tilde{h}_{\psi}(v)-1\right|^{2} S_{O O}(v) d v+\int|\tilde{d}(v)|^{2} S_{n n}(v) d v
\end{aligned}
$$

where the symbol $\sim$ refers to the Fourier transform and $v$ denotes the spatial frequency. $M S E_{\psi}$ is a sum of two terms: the first one results from the inadequacy of the deconvolution filter with the actual PSF, since the PSF varies with the defocus, and the second one is the error due the noise enhancement by the deconvolution filter.

The criterion is based on the MSE averaged over the desired defocus range $\psi_{j} \in\left[0, \psi_{\text {defoc } \max }\right]$ :

$$
M S E_{\text {mean }}=\frac{1}{n} \sum_{i=1}^{n} M S E_{\psi_{i}} .
$$

The deconvolution filter is calculated to minimize $M S E_{\text {mean }}$ :

$$
\tilde{d}(v)=\frac{\frac{1}{m} \sum_{j=1}^{m} \tilde{h}_{\psi_{j}}^{*}(v)}{\frac{1}{m} \sum_{j=1}^{m}\left|\tilde{h}_{\psi_{j}}(v)\right|^{2}+\frac{S_{n n}(v)}{S_{O O}(v)}},
$$

and depends on the object spectral content. However, the use of a generic deconvolution filter do not change significantly the image quality compared to the deconvolution filter suited for a given scene.

The proposed image quality criterion is the output signal to noise ratio (SNR), which depends on $M S E_{\text {mean }}$ calculated with $n>m$ defocus values :

$$
S N R_{\text {mean }}(d B)=10 \log _{10}\left\lfloor\int S_{O O}(v) d v / M S E_{\text {mean }}\right\rfloor .
$$

\section{Optimization of different phase masks}

Thanks to the proposed criterion, the parameters of the different phase masks are optimized, and it is possible to compare them, according to the noise of the sensor and the desired depth of focus where the imaging system should provides good image quality. In the following, we will consider a desired depth of focus $\psi \in[-10.8,10.8]$ and an input SNR $S N R_{i n}=34 d B$. The scene is the classical "Lena" image and we will investigate two types of masks.

The first phase mask which makes an optical system insensitive to defocus is the cubic phase mask [1] whose expression is $\varphi(x, y)=\alpha_{c u b}\left(x^{3}+y^{3}\right)$ with $\alpha_{c u b}$ a mask parameter, and $x$ and $y$ the coordinates of the pupil. The $S N R_{\text {mean }}$ as a function of $\alpha_{\text {cub }}$ is displayed in Fig. 1.

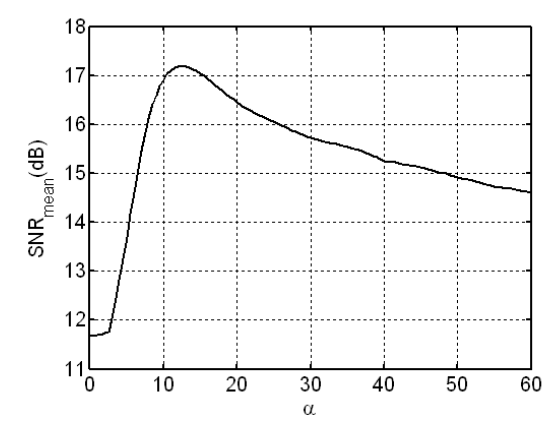

Fig. 1. $S N R_{\text {mean }}$ obtained with the cubic phase mask in function of $\alpha_{c u b}$ for a desired depth of focus $\psi \in[-10.8,10.8]$. With a conventional imaging system without postprocessing the $S N R_{\text {mean }}$ is equal to $10.8 \mathrm{~dB}$ for $\psi \in[-10.8,10.8]$ and equal to $19 \mathrm{~dB}$ for $\psi=0$

The optimal mask parameter is $\alpha_{\text {cub,opt }}=10.5$, leading to $S N R_{\text {mean }}=17.2 d B$. With lower values of $\alpha_{\text {cub }}$, the PSF of the optical system is not insensitive enough to ensure good image quality at the extrema of the desired depth of focus. With larger values of $\alpha_{c u b}$, the noise amplification due to the deconvolution leads to a lower image quality. 


\section{IMD4.pdf}

The second phase mask has an exponential profile [2] whose expression is $\varphi(x, y)=\alpha_{\exp } x^{2}[\exp (\beta x)-1]+\alpha_{\exp } y^{2}[\exp (\beta y)-1]$ with $\alpha_{\exp }$ and $\beta_{\exp }$ mask parameters. The $S N R_{\text {mean }}$ as a function of $\alpha_{\exp }$ and $\beta_{\exp }$ is displayed in Fig. 2.

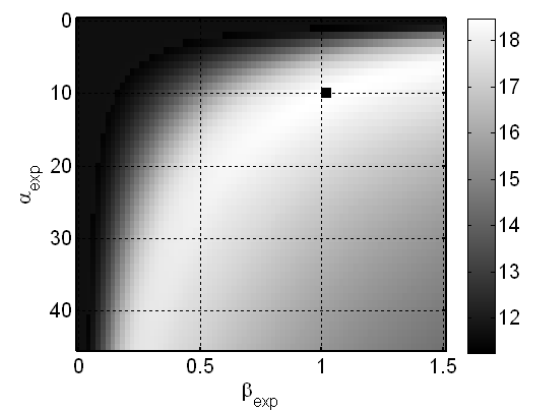

Fig. 2. $S N R_{\text {mean }}$ obtained with the exponential phase mask in function of $\alpha_{\text {exp }}$ and $\beta_{\text {exp }}$. The optimal mask parameters are represented by the square.

Optimal exponential mask parameters are $\alpha_{\text {exp }}=10$ and $\beta_{\exp }=1.04$ leading to $S N R_{\text {mean }}=18.5 d B$. According to the same $S N R_{\text {mean }}$ criterion, a better image quality can be achieved using this exponential phase mask than with the previous cubic one, as shown by the images calculated for several defocus in Fig. 3. It can be noticed that the $S N R_{\text {mean }}$ criterion is correlated with the visual perception: the exponential phase masks introduces less artefacts than the cubic one.

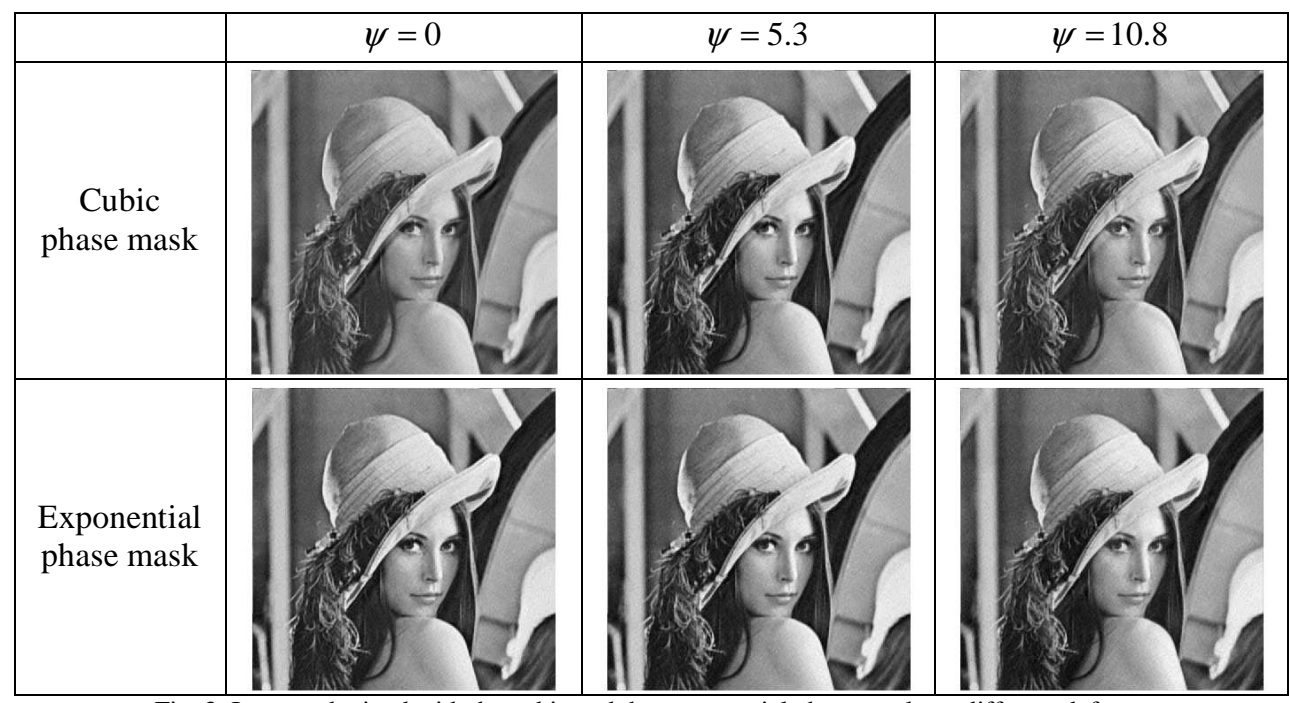

Fig. 3. Images obtained with the cubic and the exponential phase masks at different defocus

\section{Conclusion}

We defined an optimization criterion that can consider the non-invariance of the PSF and the noise enhancement induced by deconvolution process. This criterion allowed to characterize the performance of depth of focus enhancing masks, and has an effective correlation with the visual perception. So that it can be used to optimize the parameters of the masks and to compare them.

\section{References}

[1] E. R. Dowski, Jr. and W. T. Cathey, "Extended depth of field through wave-front coding', Appl. Opt. 34, 1859-1866 (1995)

[2] Q. Yang., L. Liu, J. Sun, “Optimized phase pupil masks for extended depth of field”, Opt. Commun. 272, 56-66 (2007)

[3] F. Diaz, F. Goudail, B. Loiseaux, J.-P. Huignard, "Increase in depth of field taking into account deconvolution by optimization of pupil mask", Opt. Lett. 34, 2970-2972 (2009) 